\title{
Two Constructions of Oriented Matroids with Disconnected Extension Space
}

\author{
Nicolai E. Mnëv ${ }^{1}$ and Jürgen Richter-Gebert ${ }^{2}$ \\ ${ }^{1}$ U1. Korablestroiteley 40-1-187, \\ 199155 St. Petersburg, Russia \\ ${ }^{2}$ Auf dem Wingert 3, D-64380 Roßdorf, \\ Federal Republic of Germany
}

\begin{abstract}
The extension space $\mathscr{E}(\mathscr{M})$ of an oriented matroid $\mathscr{H}$ is the poset of all one-element extensions of $\mathscr{M}$, considered as a simplicial complex. We present two different constructions leading to rank 4 oriented matroids with disconnected extension space. We prove especially that if an element $f$ is not contained in any mutation of a rank 4 oriented matroid $\mathscr{M}$, then $\mathscr{E}(\mathscr{M} \backslash f)$ contains an isolated point. A uniform nonrealizable arrangement of pseudoplanes with this property is presented.

The examples described contrast results of Sturmfels and Ziegler [12] who proved that for rank 3 oriented matroids the extension space has the homotopy type of the 2-sphere.
\end{abstract}

\section{Introduction}

Let $\mathscr{M}:=(\chi, E, d)$ be an oriented matroid of rank $d$ on the ground set

$$
E:=\{1, \ldots, n\}
$$

An oriented matroid $\mathscr{M}^{\prime}$ on $E \uplus\{f\}$ is an extension of $\mathscr{M}$ if $\mathscr{M} \backslash f=\mathscr{M}$ and $f$ is neither a loop nor a coloop in $\mathscr{M}^{\prime}$. The set of all extensions

$$
\mathscr{E}(\mathscr{M}):=\left\{\mathscr{M}^{\prime} \mid \mathscr{M}^{\prime} \backslash f=\mathscr{M}\right\}
$$

is partially ordered with $\mathscr{M}_{1}^{\prime} \leq \mathscr{M}_{2}^{\prime}$ whenever $\mathscr{M}_{1}^{\prime}$ is a weak image of $\mathscr{M}_{2}^{\prime}$ (i.e., every signed basis of $\mathscr{M}_{1}^{\prime}$ is a basis with the same sign in $M_{2}^{\prime}[2$, Section 7.7]). We can identify this poset with the simplicial complex $\Delta \mathscr{E}(\mathscr{M})$ of chains in $\mathscr{E}(\mathscr{M})$. It is 
the task of this paper to construct oriented matroids $\mathscr{M}$ such that the extension space $\Delta \mathscr{E}(\mathscr{M})$ is disconnected.

Before going into the details of the constructions we briefly collect what is known about the homotopy type of extension spaces of arrangements of oriented (pseudo) hyperplanes and oriented matroids. The first case is a central arrangement $\mathbf{X}$ of oriented hyperplanes in $\mathbb{R}^{d}$ and its space $\mathscr{E}(\mathbf{X})$ of extensions by a new oriented hyperlane $H$, partially ordered with $H \leq H^{\prime}$ whenever $H$ is in a more special position than $H$. In this case the space $\Delta \mathscr{E}(\mathrm{X})$ is homeomorphic to a sphere of dimension $(d-1)$. The situation changes drastically if we turn our attention to a more combinatorial point of view and consider the equivalence classes of oriented hyperplane arrangements induced by the underlying oriented matroids. Sturmfels and Ziegler proved that the space $\mathscr{E}_{\text {real }}(\mathscr{M})$ of all realizable extensions of a realizable oriented matroid $\mathscr{H}$ is still connected but in general does not have the homotopy type of a sphere [12].

If we consider the complete extension space $\mathscr{E}(\mathscr{M})$ of a realizable rank $d$ oriented matroid $\mathscr{M}$ (i.e., the extensions of an oriented hyperplane arrangement by a new oriented pseudoplane), the problem of determining the homotopy type of $\mathscr{E}(\mathscr{M})$ turns out to be a special case of the "Generalized Baues Problem" of Billera et al. [1]. It is conjectured that in this case $\mathscr{E}(\mathscr{M})$ has the homotopy type of a $(d-1)$-sphere.

Sturmfels and Ziegler proved that for the class of strongly euclidean oriented matroids the space $\mathscr{E}(\mathscr{M})$ has the homotopy type of a $(d-1)$-sphere [12]. The following types of oriented matroids have been proven to be strongly euclidean:

(i) $\mathscr{H}$ has rank $d \leq 3$ or corank $n-d \leq 2$.

(ii) $\mathscr{H}$ is uniform of rank 4 and has at most seven elements.

(iii) $\mathscr{H}$ is the alternating oriented matroid $C^{n, d}$ with $n$ points in rank $d$.

These results solve the "Generalized Baues Problem" for the above cases.

In this paper we present two different constructions of oriented matroids with a disconnected extension space. These are the first-known examples where the extension space is known to be nonspherical. The first construction, which is due to the first author, makes use of the fact that in a noneuclidean oriented matroid program any extension plane meeting the $L P$-cycle cannot have all infinite vertices on the same side (see [7]). A rank 4 oriented matroid $M(28)$ on 28 elements is constructed containing several copies of an 8-element noneuclidean oriented matroid such that $\mathscr{E}(M(28))$ has at least three connected components.

The second construction, which is due to the second author, is based on a rank 4 oriented matroid $R(20)$ on 20 elements where one element $f$ is not contained in any mutation (i.e., it is not adjacent to any simplicial region in the corresponding pseudoarrangement). It is proved that if for a rank 4 oriented matroid $\mathscr{M}$ the set $M u t_{f}(\mathscr{M})$ of mutations containing $f$ is empty, then $\mathscr{H}$ is an isolated point in $\mathscr{E}(\mathscr{M} \backslash f)$. A detailed description of how to construct $R(20)$ can be found in [10] where rank-preserving composition methods for oriented matroids are studied, in order to derive oriented matroids with few mutations.

As a technical tool relating the mutation structure of $\mathscr{H}$ and the topological type of $\mathscr{E}(\mathscr{M} \backslash f)$ we introduce the concept of inseparable $k$-sets. This notion 
simultaneously generalizes the concepts of mutations and inseparable pairs. We prove that $\mathscr{M}$ is an isolated element in $\mathscr{E}(\mathscr{M} \backslash f)$ if and only if $f$ is not contained in any inseparable $k$-set of $\mathscr{M}$.

\section{Noneuclideanness and Extension Spaces}

In this section we present an oriented matroid $M(28)$ whose extension space is disconnected. This example was the first-known example with this property.

We start by recalling some properties of noneuclidean oriented matroids. The concept of euclidean oriented matroids was introduced by Edmonds, Fukuda, and Mandel [7], [9]. For a detailed introduction to this topic the reader is referred to Chapter 10 of [2].

Let $\mathscr{M}$ be a rank $d$ oriented matroid on the set $E \oplus\{g\}:=\{1, \ldots, n, g\}$, where $g$ is neither a loop nor a coloop. The pair $(\mathscr{M}, g)$ is called an affine oriented matroid. As usual we identify $\mathscr{H}$ with its representation by an oriented pseudohyperplane arrangement via the Folkman and Lawrence Representation Theorem [6], [2, Theorem 5.4.1]. We interpret the distinguished pseudohyperplane $g$ as the hyperplane at infinity of an abstract affine space. The set of all covectors $Y$ of $\mathscr{M}$ with $Y_{g}=+$ is called the affine space of $(\mathscr{A}, g)$. The set of all covectors $Y$ with $Y_{g}=0$ is called the infinite space of $(\mathscr{M}, g)$. Affine cocircuits of $\mathscr{M}$ are the vertices of $(\mathscr{M}, g)$. A vertex $Y$ is said to be contained in a hyperplane $e \in E$ if $Y_{e}=0$. The extension space $\mathscr{E}(\mathscr{A}, g)$ is the subposet of extensions in $\mathscr{E}(\mathscr{M})$ which are neither parallel nor antiparallel to $g$.

Two elements $e, f \in E$ are called parallel in the affine oriented matroid $(\mathscr{M}, g)$ if they coincide at infinity (i.e., $e$ and $f$ are parallel or antiparallel elements in $\mathscr{M} / g$ ). The notion of euclideanness as introduced by Edmonds, Fukuda, and Mandel can be considered as an oriented matroid version of Euclid's Postulate of the Parallels (see [9]).

Definition 1.1. An affine oriented matroid $(\mathscr{M}, g)$ on $E \uplus\{g\}$ is called euclidean if for every element $f \in E$ and every vertex $Y$ of $(\mathscr{M}, g)$ there is an extension of $(\mathscr{M}, g)$ parallel to $f$ and containing $Y$.

By a result of Las Vergnas any extension $\mathscr{M}^{\prime} \in \mathscr{E}(\mathscr{M}, g)$ of $(\mathscr{M}, g)$ can be identified with its localization [2, Proposition 7.1.4], [8]. The localization is the unique function $\sigma_{\mathscr{M}}$ assigning to every cocircuit $Y$ of $\mathscr{M}$ a sign $\sigma_{\mathscr{M}}(Y) \in\{-, 0,+\}$ such that $\left(Y, \sigma_{\mathscr{M}}(Y)\right)$ is a cocircuit of $\mathscr{M}^{\prime}$. The sign-function $\sigma_{\mathscr{M}}$ encodes the position of the extending hyperplane with respect to the vertices of $(\mathscr{M}, g)$. The natural partial order on these localizations induced by " $0<+$ " and " $0<-$ " is isomorphic to the partial order of the extensions by weak image.

In what follows it is useful to consider euclideanness only with respect to a special element $f$ of the oriented matroid. Therefore we introduce the notion of oriented matroid programs (see [2, Definition 10.1.3], [3], and [7]). An oriented matroid program is a triple $(\mathscr{H}, g, f)$ where $\mathscr{M} \in \mathscr{E}(\mathscr{M} \backslash f, g)$ is an extension of 
$(\mathscr{M}, g)$ on the ground set $E \uplus\{f, g\}$. The oriented matroid program $(\mathscr{M}, f, g)$ is euclidean if for every vertex $Y$ of $(\mathscr{A} \backslash f, g)$ there is an extension in $\mathscr{E}(\mathscr{M}, g)$ containing $Y$ and parallel to $f$.

According to Bland [3] we can consider an oriented matroid program $(\mathscr{M}, g, f)$ as an oriented matroid version of a linear programming problem. Here the hyperplane $f$ plays the role of an objective function. Following [7] and [12] we define the (partially) oriented graph $G_{(\mu, g, \Omega)}$ of an oriented matroid program $(\mathscr{M}, g, f)$ as follows. The vertices of $G_{(\mathscr{H}, g, f)}$ are the vertices of $(\mathscr{M} \backslash f, g)$. The edges of $G_{(\mathscr{M}, g, f)}$ are those pairs $\left(Y^{1}, Y^{2}\right)$ of affine cocircuits of $(\mathscr{M} \backslash f, g)$ that differ on exactly two elements $a, b \in E$ where $Y_{a}^{1} \neq 0, Y_{a}^{2}=0$ and $Y_{b}^{2} \neq 0, Y_{b}^{1}=0$. The edges of $G_{(\mathscr{M}, g, f)}$ are in one-to-one correspondence with the affine 1-cells of the pseudoarrangement of $(\mathscr{M} \backslash f, g)$. For an edge $\left(Y^{1}, Y^{2}\right)$ of $G_{(\mathscr{M}, g, f)}$ the direction from $Y^{1}$ to $Y^{2}$ is the unique infinite cocircuit $d\left(Y^{1} \rightarrow Y^{2}\right)$ of $\mathscr{M} \backslash f$ that is obtained by elimination of $g$ from $Y^{1}$ and $-Y^{2}$. The edge $\left(Y^{1}, Y^{2}\right)$ of $G_{(\mathscr{\mu}, g, f)}$ is directed from $Y^{1}$ to $Y^{2}$ if and only if $d\left(Y^{1} \rightarrow Y^{2}\right)_{f}=+$. If $d\left(Y^{1} \rightarrow Y^{2}\right)_{f}=0$, then $\left(Y^{1}, Y^{2}\right)$ remains undirected. We write $Y^{1} \preceq Y^{2}$ if $\left(Y^{1}, Y^{2}\right)$ is either undirected or directed from $Y^{1}$ to $Y^{2}$. We can consider $G_{(\mathscr{k}, g, f)}$ as a representation of the affine part of the one-skeleton of the pseudoarrangement of the affine oriented matroid $(\mathscr{M} \backslash f, g)$. The directions of the edges of $G_{(\mathscr{M}, g, \delta)}$ model the increase of a (pseudo-) linear objective function whose level set is given by $f$. Notice that $G_{(, \mathscr{H}, g, f)}$ is completely determined by $(\mathscr{M} \backslash f, g)$ and the contraction $\mathscr{M} / g$ which encodes the behavior of $f$ at infinity. A cycle $Y^{1} \preceq Y^{2} \preceq \cdots \preceq Y^{k} \preceq Y^{1}$ in $G_{(\mathscr{M}, g, f)}$ that contains at least one directed edge is called a directed cycle of $G_{(\mathscr{M}, g, f)}$.

If $\mathscr{M}$ is realizable the graph $G_{(\mathscr{K}, g, f)}$ cannot contain a directed cycle. This is no longer true for the nonrealizable case. According to Fukuda [7] and Mandel [9] the nonexistence of cycles in $G_{(\mathscr{M}, g, f)}$ can be used as an intrinsic characterization of euclideanness.

Theorem 1.2 (Fukuda, Mandel). An oriented matroid program $(\mathscr{M}, g, f)$ is euclidean if and only if the graph $G_{(\mathscr{M}, g, f)}$ does not contain a directed cycle.

The existence of directed cycles in $G_{(\mathscr{M}, g, f)}$ restricts the possible locations of $f$ in $\mathscr{M}$ as shown by the following lemma. A proof of this result can be found in Section 10.5 of [2].

Lemma 1.3. Let $(\mathscr{M}, g, f)$ be a noneuclidean oriented matroid program and let $Y^{1} \preceq Y^{2} \preceq \cdots \preceq Y^{k} \preceq Y^{1}$ be a directed cycle in $G_{(\mathscr{M}, g, f)}$, then we have either $Y_{f}^{1}=\cdots=Y_{f}^{k}=+$ or $Y_{f}^{1}=\cdots=Y_{f}^{k}=-$.

In other words, all vertices of a directed cycle lie strictly on the same side of $f$. This is the property of a noneuclidean oriented matroid which is important in connection with our extension-space problem.

Before describing the concrete oriented matroid $M(28)$ we present the main idea of this example as a lemma that guarantees the disconnectedness of the extension spaces for a whole class of oriented matroids. 
Theorem 1.4. Let $\mathscr{M}$ be an oriented matroid of rank 4 on $E \uplus\left\{g_{1}, g_{2}, g_{3}, g_{4}, f\right\}$ such that $\left(g_{1}, \ldots, g_{4}\right)$ is a basis and the oriented matroid programs $\left(\mathscr{M}, g_{i}, f\right)$ for $i=1, \ldots, 4$ are noneuclidean and have the following properties:

(i) The graph $G_{\left(\mathscr{H}, g_{i}, f\right)}$ contains a directed cycle

$$
Y^{1, i} \preceq \cdots \preceq Y^{k_{, i} i} \preceq Y^{k_{i}+1, i}=Y^{1, i}
$$

(ii) $Y_{g_{i}}^{j, i}=-Y_{j}^{j, i}=-$ for all $j=1, \ldots, k_{i}$ and $l \in\{1, \ldots, 4\} \backslash\{i\}$.

(iii) $d\left(Y^{j, i} \rightarrow Y^{j+1, i}\right)_{g_{i}}=d\left(Y^{j, i} \rightarrow Y^{j+1, i}\right)_{f}=+$ for all $j=1, \ldots, k_{i}$ and

$$
l \in\{1, \ldots, 4\} \backslash\{i\} .
$$

(iv) $\mathscr{M}$ contains the circuit $C:=\left(C^{+}, C^{-}\right)=\left(\left\{g_{1}, \ldots, g_{4}\right\},\{f\}\right)$.

In this case $\mathscr{E}(\mathscr{M} \backslash f)$ is disconnected.

Proof. Clearly, $\mathscr{M}$ as well as $-f^{\mathscr{M}}$ are extensions of $\hat{\mathscr{M}}:=\mathscr{M} \backslash f$. Here $-f^{\mathscr{M}}$ represents the oriented matroid obtained from $\mathscr{M}$ by reversing the signs of all bases containing $f$. For the corresponding localizations in $\mathscr{E}(\hat{\mathscr{M}})$ we obtain $\sigma_{\mathscr{A}}=-\sigma_{-f \mathscr{M}}$. If $\mathscr{E}(\hat{\mathscr{M}})$ is connected, then there is a sequence of extensions,

$$
\sigma_{\mathscr{M}}=\sigma_{0}, \sigma_{1}, \ldots, \sigma_{s}=-\sigma_{\mathscr{M}},
$$

such that for every $i \in\{1, \ldots, s-1\}$ either $\sigma_{i} \prec \sigma_{i+1}$ or $\sigma_{i} \succ \sigma_{i+1}$ in the extensionposet of $\hat{\mathscr{M}}$. The existence of such a sequence simply encodes the different steps of a continuous deformation of $\sigma_{\mathscr{M}}$ to $-\sigma_{\mathscr{M}}$. We prove that the existence of such a sequence contradicts conditions (i)-(iv) of our theorem.

Condition (iv) implies that $\mathscr{M} \backslash E$ contains covectors $\Delta, \Delta^{1}, \ldots, \Delta^{4}, \delta^{1}, \ldots, \delta^{4}$ defined by

$$
\begin{aligned}
\left(\Delta^{+}, \Delta^{-}\right) & :=\left(\left\{f, g_{1}, \ldots, g_{4}\right\}, \varnothing\right), \\
\left(\left(\Delta^{i}\right)^{+},\left(\Delta^{i}\right)^{-}\right) & :=\left(\left\{f, g_{i}\right\},\left\{g_{1}, \ldots, \hat{g}_{i}, \ldots, g_{4}\right\}\right), \\
\left(\left(\delta^{i}\right)^{+},\left(\delta^{i}\right)^{-}\right) & :=\left(\left\{f, g_{1}, \ldots, \hat{g}_{i}, \ldots, g_{4}\right\}, \varnothing\right) .
\end{aligned}
$$

These covectors correspond to cells of the pseudoarrangement of $\mathscr{M} \backslash E$. Namely, $\Delta$ corresponds to the positive simplicial cell bounded by $g_{1}, \ldots, g_{4}$. The $\delta^{i}$ are the 2-faces of $\Delta$ incident with $g_{i}$. Finally, the $\Delta^{i}$ are the four full-dimensional simplicial cells containing exactly one vertex of $\Delta$. We refer to $\mathscr{M} \backslash E$ as the frame of $\mathscr{M}$. The above cells can be found as supercells (see [9]) in the pseudoarrangement of $\mathscr{M}$ (i.e., they are cells of a suitable deletion). Condition (ii) of the theorem states that for $i \in\{1, \ldots, 4\}$ the vertices $Y^{1, i}, \ldots, Y^{k_{i .} i}$ are contained in the supercell $\Delta^{i}$. Condition (iii) states that for $i \in\{1, \ldots, 4\}$ in the oriented matroid program $\left(\mathscr{K}, g_{i}, f\right)$ the directions $d\left(Y^{1, i} \rightarrow Y^{2, i}\right), \ldots, d\left(Y^{k_{i, i}} \rightarrow Y^{1, i}\right)$ are contained 


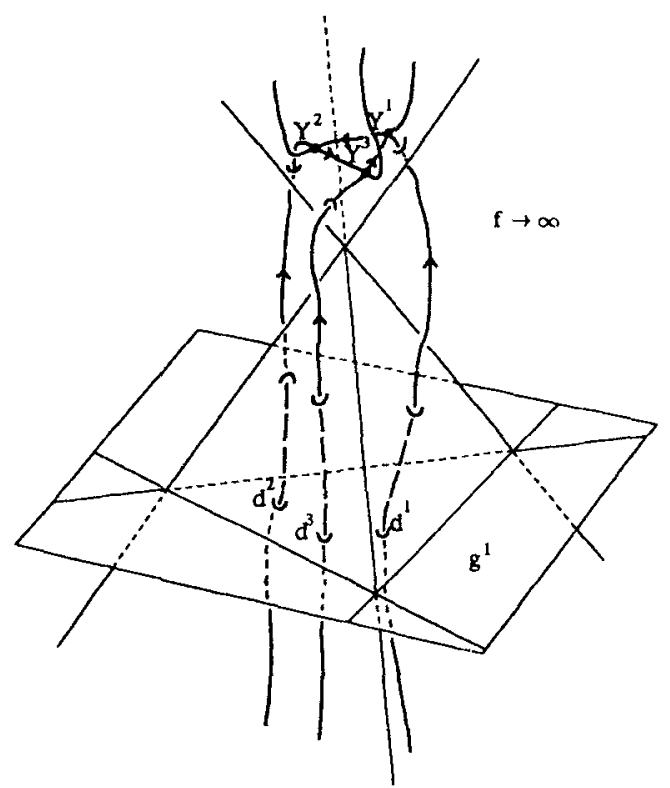

Fig. 1. Geometric situation for the oriented matroid program $\left(\mathscr{A}, g_{1}, f\right)$.

in the supercells $\delta^{i}$. We abbreviate $d^{j, i}:=d\left(Y^{j, i} \rightarrow Y^{j+1, i}\right)$ for $i \in\{1, \ldots, 4\}$ and $j \in\left\{1, \ldots, k_{i}\right\}$. Figure 1 shows the geometric situation for the case $i=1$. Here the planes forming the big tetrahedron together with plane $f$ located at infinity correspond to the frame of the configuration. The locations of the points $d^{j .1}$ and $Y^{j, i}$ are indicated in the figure.

We now consider the sets of cocircuits

$$
\mathbf{Y}:=\left\{Y^{i, j} \mid 1 \leq i \leq 4 ; 1 \leq j \leq k_{i}\right\} \quad \text { and } \quad \mathbf{d}:=\left\{d^{i, j} \mid 1 \leq i \leq 4 ; 1 \leq j \leq k_{i}\right\}
$$

By definition we have $Y_{f}=+$ for all $Y \in Y \cup d$. Hence for all $Y \in Y \cup d$ we obtain $\sigma_{0}(Y)=\sigma_{\mathscr{M}}(Y)=+$. There is a smallest index $r$ in the sequence $(*)$ such that there exists a cocircuit $Y^{\prime} \in Y \cup d$ with $\sigma_{r}\left(Y^{\prime}\right)=0$. In this case we have $\sigma_{r}(Y) \geq 0$ for every cocircuit $Y \in \mathbf{Y} \cup \mathbf{d}$. We prove that $Y^{\prime}$ can neither belong to $Y$ nor to d. This gives the desired contradiction. The oriented matroid corresponding to $\sigma_{r}$ is denoted by $\mathscr{M}^{\prime}:=\hat{\mathscr{M}} \cup f^{\prime} \in \mathscr{E}(\hat{\mathscr{M}})$.

First assume that the set $\left\{Y \in \mathbf{d} \mid \sigma_{r}(Y)=0\right\}$ is not empty. Then the extension hyperplane $f^{\prime}$ of $\hat{\mathscr{M}}$ corresponding to $\sigma_{r}$ intersects the boundary of the supercell $\Delta$. In this case the hyperplane $f^{\prime}$ has at least one supercell $\Delta^{i}$ on its negative side which contradicts the fact $\sigma_{r}(\mathbf{Y}) \geq 0$.

Now assume that $\left\{Y \in \mathrm{d} \mid \sigma_{r}(Y)=0\right\}=\varnothing$ and that $\sigma_{r}\left(Y^{j, i}\right)=0$ for suitable indices $i \in\{1, \ldots, 4\}$ and $j \in\left\{1, \ldots, k_{i}\right\}$. We consider the oriented matroid program $\left(\mathscr{M}^{\prime}, f^{\prime}, g_{i}\right)$. We have $d_{f^{\prime}}^{1, i}=\cdots=d_{f^{\prime}}^{k_{j}, i}=+$. Furthermore, we know that the sequence $Y^{1, i}, \ldots, Y^{\boldsymbol{k}_{1, i} i}$ is a directed cycle in $G_{\left(\mathcal{M}, g_{i}, f\right)}$ by condition (i). Hence the 
sequence $Y^{1, i}, \ldots, Y^{k_{1, i}}$ is also a directed cycle in $G_{\left(\mathcal{M}^{\prime}, g_{i}, s^{\prime}\right)}$. By Lemma 1.3 we obtain $Y_{f^{\prime}}^{j, i} \neq 0$ for all $j \in\left\{1, \ldots, k_{i}\right\}$. This contradicts our assumption $\sigma_{r}\left(Y^{j, i}\right)=0$. This proves the theorem.

We now present an oriented matroid $\mathscr{M}:=M(28) \cup f$ on 29 elements fulfilling conditions (i)-(iv) of Theorem 1.4. We describe the construction by explicitly giving coordinates of an oriented matroid $\mathscr{M}^{\prime}$ on 29 elements that after a suitable perturbation becomes $\mathscr{M}$. The arrangement $\mathscr{M}^{\prime}$ mainly consists of two basic subconfigurations. The first subconfiguration is the frame $\left(f, g_{1}, g_{2}, g_{3}, g_{4}\right)$ of $\mathscr{M}^{\prime}$ located at

$$
F:=\left(\begin{array}{ccccc}
f & g_{1} & g_{2} & g_{3} & g_{4} \\
1 & 1 & 0 & 0 & 0 \\
1 & 0 & 1 & 0 & 0 \\
1 & 0 & 0 & 1 & 0 \\
1 & 0 & 0 & 0 & 1
\end{array}\right)
$$

The second subconfiguration of $\mathscr{M}^{\prime}$ is given by the matrix

$$
C_{1}:=\left(\begin{array}{rrrrrr}
1 & 2_{1} & \multicolumn{1}{c}{3_{1}} & \multicolumn{1}{c}{4_{1}} & \multicolumn{1}{c}{5_{1}} & \multicolumn{1}{c}{6_{1}} \\
100 & 100 & 100 & 98 & 98 & 98 \\
-601 & 100 & 101 & -600 & 99 & 110 \\
101 & -601 & 100 & 110 & -600 & 99 \\
100 & 101 & -601 & 99 & 110 & -600
\end{array}\right):=\left(\begin{array}{l}
r_{1} \\
r_{2} \\
r_{3} \\
r_{4}
\end{array}\right)
$$

Symmetrically we define arrangements $C_{2}, C_{3}, C_{4}$ by rotating the rows of the matrix $C_{1}$ :

$$
\begin{array}{ll}
C^{2}:=\left(r_{4}, r_{1}, r_{2}, r_{3}\right)^{T} & \text { on }\left\{1_{2}, \ldots, 6_{2}\right\}, \\
C^{3}:=\left(r_{3}, r_{4}, r_{1}, r_{4}\right)^{T} & \text { on }\left\{1_{3}, \ldots, 6_{3}\right\}, \\
C^{4}:=\left(r_{2}, r_{3}, r_{4}, r_{1}\right)^{T} & \text { on }\left\{1_{4}, \ldots, 6_{4}\right\}
\end{array}
$$

Define $\mathscr{M}^{\prime}$ to be the oriented matroid corresponding to the hyperplane arrangement $\left(F, C_{1}, C_{2}, C_{3}, C_{4}\right)$.

The hyperplanes corresponding to $C_{i}$ describe the faces of a small cube located in the simplicial cell $\Delta_{i}$ of the frame $F$ bounded by $f, g_{1}, \ldots, \hat{g}_{i}, \ldots, g_{4}$. The coordinates are chosen in such a way that all intersections of six planes of $C_{i}$ also lie in $\Delta^{i}$ and such that all lines that are the intersections of two planes in $C_{i}$ meet the plane $g_{i}$ in the region $\delta^{i}$ bounded by $g_{1}, \ldots, \hat{g}_{i}, \ldots, g_{4}$. Furthermore, the quadruples $\left(1_{i}, 2_{i}, 4_{i}, 5_{i}\right),\left(1_{i}, 3_{i}, 4_{i}, 6_{i}\right)$, and $\left(2_{i}, 3_{i}, 5_{i}, 6_{i}\right)$ bound tetrahedral regions (mutations) in the hyperplane arrangement $\left(F, C_{i}\right)$. If we perturb these three simplicial regions in the corresponding oriented matroid $\mathscr{M}_{i}^{\prime}$ we end up with an 
oriented matroid $\mathscr{M}_{i}$ on $\left(f, g_{1}, \ldots, g_{4}, 1_{i}, \ldots, 6_{i}\right)$ such that $\left(\mathscr{M}_{i} \backslash\left\{g_{1}, \ldots, \hat{g}_{i}, \ldots, g_{4}\right\}\right.$, $g_{1}, f$ ) is noneuclidean (namely, the underlying oriented matroid is isomorphic to the unique example with eight elements and only seven mutations (see [4] and [11])). The vertices of the corresponding cycle all lie in $\delta^{i}$.

We define $\mathscr{M}$ on $\left\{f, g_{1}, \ldots, g_{4}, 1_{1}, \ldots, 6_{4}\right\}$ by its basis orientations:

$$
\chi_{\mathscr{M}}(\lambda):= \begin{cases}-1 & \text { if } \lambda \in\left\{\left(1_{i}, 2_{i}, 4_{i}, 5_{i}\right),\left(1_{i}, 3_{i}, 4_{i}, 6_{i}\right),\left(2_{i}, 3_{i}, 5_{i}, 6_{i}\right) \mid 1 \leq i \leq 4\right\} \\ \chi_{\mathscr{M}}(\lambda) & \text { otherwise. }\end{cases}
$$

By our above observations $\mathscr{M}$ fulfills the requirements to apply Theorem 1.4. We define $M(28):=\mathscr{M} \backslash f$ and obtain:

Theorem 1.5. The extension space $\mathscr{E}(M(28))$ is not connected.

Remark 1.6. In fact the extension space $\mathscr{E}(M(28))$ has at least three connected components since $\mathscr{M},-f \mathscr{M}$, and any extension of $M(28)$ parallel to any other element lie in different connected components.

\section{Mutations and Extension Spaces}

In this section we present a second way to generate an oriented matroid with disconnected extension space. We therefore clarify the connections between the extension space of an oriented matroid and the structure of its mutations (see Section 7.3 of [2]). In order to do this we introduce the more general concept of inseparable $k$-sets, which simultaneously generalizes the concepts of mutations and the concept of inseparable pairs. We frequently identify an oriented matroid $\mathscr{M}$ with its chirotope $\chi:=\chi_{\mathscr{\mu}}$. We set

$$
\Lambda(E, d):=\left\{\left(\lambda_{1}, \ldots, \lambda_{d}\right) \in E^{d} \mid \lambda_{1}<\cdots<\lambda_{d}\right\}
$$

and define mutations by:

Definition 2.1. Let $\chi: \Lambda(E, d) \rightarrow\{-1,+1\}$ be a uniform oriented matroid of rank $d$. A basis $\lambda \in \Lambda(E, d)$ is called a mutation if the map defined by

$$
\chi^{-\lambda}\left(\lambda^{\prime}\right):= \begin{cases}\chi\left(\lambda^{\prime}\right) & \text { for } \lambda \neq \lambda^{\prime} \\ -\chi\left(\lambda^{\prime}\right) & \text { for } \lambda=\lambda^{\prime}\end{cases}
$$

is again an oriented matroid.

Thus the mutations of an oriented matroid correspond to the possibilities of local modifications of the basis orientations of $\mathscr{M}$. The set of all mutations of an oriented matroid $\mathscr{M}$ is denoted by $M u t(\mathscr{M})$. The set of all mutations containing 
a given element $f \in E$ is denoted by $M u t_{f}(\mathscr{M})$. There are many equivalent ways to characterize mutations; some of them are summarized in the following lemma.

Lemma 2.2. Let $\chi$ be a uniform oriented matroid of rank $d$ on a finite set $E=\{1, \ldots, n\}$ and let $\lambda \in \Lambda(E, d)$. The following statements are equivalent:

(i) $\lambda$ is a mutation.

(ii) $\lambda$ forms a simplicial cell in the pseudohyperplane arrangement $H_{\mathscr{A}}$ corresponding to $\mathscr{M}$.

(iii) The restriction of the cocircuit graph on $\mathscr{M}$ to the vertex set $\Lambda(\lambda, d-1)$ is the complete graph $K_{d}$.

(iv) The restriction of the cocircuit graph to the vertex $\operatorname{set} \Lambda(\lambda, d-1)$ is connected.

(v) For every decomposition $\tau_{1} \uplus \tau_{2}=\lambda$ the $k$-tuple $\tau_{1}$ is a mutation in $\chi / \tau_{2}$.

(vi) $\lambda$ is not determined by any Grassmann-Plücker relation (see [11]).

Proof. Characterization (ii) can be found in [11]. Characterization (iii) follows directly from (ii). Characterization (iv) is a reformulation of the fact that a mutation $\left(\lambda_{1}, \ldots, \lambda_{d}\right)$ forms a simplex in the pseudoarrangement that is not cut by any other hyperplane. Characterizations (v) and (vi) can be found in Section 5.3 of [2].

The following lemma summarizes results concerning lower bounds on the number of mutations of euclidean oriented matroids. Lemma 2.3, especially, applies to all realizable oriented matroids and to all oriented matroids of at most rank 3.

Lemma 2.3 (see [11]). Let $\mathscr{M}$ be a uniform oriented matroid of rank $d$ on a finite set $E=\{1, \ldots, n\}$ and let $f \in E$. If $\mathscr{M}$ is either realizable or $d \leq 3$, then $\mid$ Mut $_{f}(\mathscr{M}) \mid \geq d$ and $|M u t(\mathscr{M})|-\left|M u t_{f}(\mathscr{M})\right| \geq n-d$.

We now introduce the concept of inseparable $k$-sets of a uniform oriented matroid. They are "mutation-like" structures of dimension $k$.

Definition 2.4. Let $\mathscr{M}$ be a uniform rank $d$ oriented matroid on $E$. An element $\lambda \in \Lambda(E, k)$ is called an inseparable $k$-set if $\lambda \in M u t(\mathscr{M} / \tau)$ for all $\tau \in \Lambda(E-\lambda, d-k)$.

Remark 2.5. According to this definition usual mutations of a rank $d$ oriented matroid correspond to inseparable $d$-sets and inseparable pairs correspond to inseparable 2-sets.

We abbreviate the set of all inseparable $k$-sets of a given oriented matroid $\mathscr{M}$ by $\operatorname{Mut}^{k}(\mathscr{H})$. The set of all inseparable $k$-sets containing a given element $f \in E$ is abbreviated by $M u t_{f}^{k}(\mathscr{M})$. 
If $\mathscr{M}$ is a uniform oriented matroid of either rank 2 or rank 3 , then by Lemma 2.3 there is always a mutation that contains a given element $f$. This translates to the following more general statement.

Lemma 2.6. Let $\mathscr{M}$ be a uniform oriented matroid of rank $d$ on a finite set $E$ and let $f \in E$. Then we have:

(i) $M u t_{f}^{d-2}(\mathscr{M}) \neq \varnothing \Rightarrow M u t_{f}^{d}(\mathscr{M})=M u t_{f}(\mathscr{M}) \neq \varnothing$.

(ii) $M u t_{f}^{d-1}(\mathscr{M}) \neq \varnothing \Rightarrow M u t_{f}^{d}(\mathscr{M})=M u t_{f}(\mathscr{M}) \neq \varnothing$.

Proof. (i) Assume that $\left(\lambda_{1}, \ldots, \lambda_{d-2}\right) \in M u t_{f}^{d-2}(\mathscr{M})$. For the rank 3 oriented matroid $\mathscr{M}_{3}:=\mathscr{M} /\left\{\lambda_{1}, \ldots, \lambda_{d-3}\right\}$ by Lemma 2.3 there is a mutation

$$
\left(\lambda_{d-2}, \lambda_{d-1}, \lambda_{d}\right) \in M u t_{\lambda_{d-2}}^{3}\left(\mathscr{M}_{3}\right)
$$

that contains $\lambda_{d-2}$. We now prove that $\left(\lambda_{1}, \ldots, \lambda_{d}\right)$ is a mutation of $\chi$. Remember that the cocircuit graph $C G(\mathscr{M})$ of $\mathscr{M}$ corresponds to the one-skeleton of the corresponding pseudoarrangement. By Lemma 2.2 it is sufficient to show that the restriction $C G^{\lambda}$ of the cocircuit graph $C G(\mathscr{M})$ of $\mathscr{M}$ to the vertices

$$
C_{i}:=\left(\lambda_{1}, \ldots, \hat{\lambda}_{i}, \ldots, \lambda_{d}\right)
$$

for $i \in\{1, \ldots, d\}$ is connected. The fact that $\left(\lambda_{1}, \ldots, \lambda_{d-2}\right) \in M u t_{f}^{d-2}(\mathscr{M})$ implies the connectedness of $C_{1}, \ldots, C_{d-2}$ in $C G^{\lambda}$. The fact that $\left(\lambda_{d-2}, \lambda_{d-1}, \lambda_{d}\right) \in M u t_{\lambda_{d-2}}^{3}\left(\mathscr{M}_{3}\right)$ implies the connectedness of $C_{d-2}, \ldots, C_{d}$ in $C G^{\lambda}$. This proves the claim.

(ii) The proof is analogous to the one given for case (i). The only difference is in the dimension and ranks of the objects considered.

The following result connects the inseparable $k$-sets $M u t_{f}^{k}(\mathscr{M})$ to the extension space of $\mathscr{M} \backslash f$ :

Theorem 2.7. Let $\mathscr{M}$ be a uniform oriented matroid on $E$ and let $f \in E$. The following statements are equivalent:

(i) $M u t_{f}^{k}(\mathscr{M})=\varnothing$ for all $k \in\{2, \ldots, d\}$.

(ii) The extension $\mathscr{M}$ is an isolated point in the extension space $\mathscr{E}(\mathscr{M} \backslash f)$.

Proof. Not (i) $\Rightarrow$ not (ii). We set $\chi:=\chi_{\mu}$. Assume that there is a $k \in\{2, \ldots, d\}$ with $M u t_{f}^{k}(\mathscr{K}) \neq \varnothing$ and let $\lambda:=\left(\lambda_{1}, \ldots, \lambda_{k}\right) \in M u t_{f}^{k}(\mathscr{M})$. We prove that the map $\tilde{\chi}$ defined by

$$
\tilde{\chi}\left(\lambda^{\prime}\right):= \begin{cases}\chi\left(\lambda^{\prime}\right) & \text { for } \lambda \nsubseteq \lambda^{\prime}, \\ 0 & \text { for } \lambda \subseteq \lambda^{\prime}\end{cases}
$$


again forms an oriented matroid which is a nontrivial extension of $\mathscr{M} \backslash f$. The only statement we have to prove is that $\tilde{\chi}$ is again an oriented matroid. This can be done by showing that all three-summand Grassmann-Plücker relations are fulfilled (see [11], [5], and [2, Theorem 3.6.2]) and showing that $\tilde{M}:=\left\{(b) \in E^{d} \mid \tilde{\chi}(b) \neq 0\right\}$ forms the set of bases of a matroid. The second condition is fulfilled trivially since $\tilde{M}$ is a matroid with just one dependent set $\lambda$. Following [5] we now interpret the three summand Grassmann-Plücker relations as polynomials over the fuzzy-ring of $G F^{3}$. The Grassmann--Plücker relations of the form

$$
\begin{aligned}
& \{\lambda, \tau \mid, a, b, c, f\}_{\tilde{\chi}}= \\
& \quad \underbrace{[\tilde{\chi}(\lambda, \tau, a, b)}_{=0} \underbrace{\tilde{\chi}(\lambda, \tau, c, f)}_{=0}-\underbrace{\tilde{\chi}(\lambda, \tau, a, c)}_{=0} \underbrace{\tilde{\chi}(\lambda, \tau, b, f)}_{=0}-\underbrace{\tilde{\chi}(\lambda, \tau, a, f)}_{=0} \underbrace{\tilde{\chi}(\lambda, \tau, b, c)}_{=0} \in\{0, *\}],
\end{aligned}
$$

where $\tau \in \Lambda(E-\lambda, d-k-2)$ and $a, b, c, d \in E-\lambda-\tau$ are satisfied since here all summands turn out to be zero. The same holds for the Grassmann-Plücker relations of the form

$$
\left\{\lambda_{1}, \ldots, \hat{\lambda}_{i}, \ldots, \lambda_{k}, \tau \mid, a, b, c, \lambda_{i}\right\}_{\tilde{\chi}}
$$

where $\tau \in \Lambda(E-\lambda, d-k-1), i \in\{1, \ldots, k\}$, and $a, b, c \in E-\lambda-\tau$. The remaining Grassmann-Plücker relations are all fulfilled by Lemma $2.2($ vi) and the fact that for every $\tau \in \Lambda(E-\lambda, d-k)$ the inseparable $k$-set $\lambda$ forms a mutation in $\chi / \tau$.

(i) $\Rightarrow$ (ii) We subdivide the proof of this part of the statement into several claims. In order to simplify the notations we abbreviate, for $\lambda \in \Lambda(E, k)$ and $k \leq d$,

$$
\chi(\lambda)=0 \Leftrightarrow[\chi(\lambda, \tau)=0 \text { for all } \tau \in \Lambda(E-\lambda, d-k)]
$$

In what follows let $\chi^{\prime}$ be the chirotope of a (not necessarily uniform) extension in $\mathscr{E}(\mathscr{M} \backslash f)$. Since $\mathscr{M} \backslash f$ is uniform we have $\chi^{\prime}(\lambda) \neq 0$ for all $\lambda \in \Lambda(E, d)$ with $f \notin \lambda$.

Claim 1. For $\lambda \in \Lambda(E-\{f\}, k)$ with $k \leq d$ and different elements $a, b \in E-\lambda$ we have

$$
\left[\chi^{\prime}(f, \lambda, a)=0 \text { and } \chi^{\prime}(f, \lambda, b)=0\right] \quad \Rightarrow \quad \chi^{\prime}(f, \lambda)=0 \text {. }
$$

Proof. We have to prove that $\chi^{\prime}(\lambda, c)=0$ for all $c \in E-\lambda-\{f, a, b\}$. Consider $\tau \in \Lambda(E-\lambda-\{f, a, b\}, d-k-2)$. The Grassmann-Plücker relation

$$
\begin{aligned}
& \{\lambda, \tau \mid a, b, c, f\}= \\
& \underbrace{\left[\chi^{\prime}(\lambda, \tau, a, b)\right.}_{=0} \chi^{\prime}(\lambda, \tau, c, f)-\underbrace{\chi^{\prime}(\lambda, \tau, a, c)}_{=0} \underbrace{\chi^{\prime}(\lambda, \tau, b, f)}_{=0}-\underbrace{\chi^{\prime}(\lambda, \tau, a, f}_{\neq 0}) \underbrace{\chi^{\prime}(\lambda, \tau, b, c)}_{=0} \in\{0, *\}]
\end{aligned}
$$

implies that $\chi^{\prime}(\lambda, \tau, c, f)=0$. This proves the claim. 
Claim 2. For $\lambda \in \Lambda(E-\{f\}$, $k)$ with $k \leq d$ and $l+1 \leq d-k$ pairwise different elements $a, b_{1}, \ldots, b_{l} \in E-\lambda$ we have

$$
\left[\chi^{\prime}(f, \lambda, a)=0 \text { and } \chi^{\prime}\left(f, \lambda, b_{1}, \ldots, b_{l}\right)=0\right] \quad \Rightarrow \quad \chi^{\prime}(f, \lambda)=0 \text {. }
$$

Proof. This result is simply a consequence of the inductive application of Claim 1. The case $l=1$ is covered by Claim 1 . Now assume that the

$$
\left[\chi^{\prime}(f, \lambda, a)=0 \text { and } \chi^{\prime}\left(f, \lambda, b_{1}, \ldots, b_{l-1}\right)=0\right] \quad \Rightarrow \quad \chi^{\prime}(f, \lambda)=0
$$

is proved. Then we have

$$
\begin{aligned}
& {\left[\chi^{\prime}(f, \lambda, a)=0 \text { and } \chi^{\prime}\left(f, \lambda, b_{1}, \ldots, b_{l}\right)=0\right]} \\
& \quad \Rightarrow \quad\left[\chi^{\prime}(f, \lambda, a)=0 \text { and } \chi^{\prime}\left(f, \lambda, a, b_{1}, \ldots, b_{l-1}\right)=0 \text { and } \chi^{\prime}\left(f, \lambda, b_{1}, \ldots, b_{l}\right)=0\right] \\
& \quad \Rightarrow \quad\left[\chi^{\prime}(f, \lambda, a)=0 \text { and } \chi^{\prime}\left(f, \lambda, b_{1}, \ldots, b_{l-1}\right)=0\right] \\
& \quad \Rightarrow \quad \chi^{\prime}(f, \lambda) .
\end{aligned}
$$

This proves Claim 2 .

Claim 3. For $\lambda \in \Lambda(E-f, k)$ where $k \leq d$ we have

$$
\begin{aligned}
& {\left[\chi^{\prime}(f, \lambda)=0 \text { and }(f, \lambda) \notin M u t_{f}^{k+1}(\mathscr{M})\right]} \\
& \quad \Rightarrow \text { there exists an } i \in\{1, \ldots, k\} \text { such that } \chi^{\prime}\left(f, \lambda_{1}, \ldots, \hat{\lambda}_{i}, \ldots, \lambda_{k}\right)=0 .
\end{aligned}
$$

Proof. If $(f, \lambda) \notin M u t_{f}^{k+1}(\mathscr{A})$, then there is a $\tau \in \Lambda(E-\lambda-\{f\}, d-k-1)$ such that $(f, \lambda)$ is no mutation in $\chi / \tau$. In this case by Lemma 2.2 (iv) and (vi) there are $a, b \in E-\tau-\lambda-\{f\}$ and $i \in\{1, \ldots, k\}$ such that the basis $(\tau, \lambda, f)$ is not determined by the Grassmann-Plücker relation $\left\{\tau, \lambda_{1}, \ldots, \hat{\lambda}_{i}, \ldots, \lambda_{k} \mid f, \lambda_{i}, a, b\right\}$. Without loss of generality we may assume that $i=k$. If $\chi^{\prime}(f, \lambda)=0$ we have, especially, $\chi^{\prime}(\tau, \lambda, f)=0$. The above Grassmann-Plücker relation implies that in this case we also obtain $\chi^{\prime}\left(\tau, \lambda_{1}, \ldots, \lambda_{k-1}, f, a\right)=0$. Now Claim 2 together with $\chi^{\prime}(f, \lambda)=0$ implies $\chi^{\prime}\left(f, \lambda_{1}, \ldots, \lambda_{k-1}\right)=0$. This proves Claim 3 .

We are now ready to complete the proof of Theorem 2.7. It remains to show that under the assumptions that $M u t_{f}^{k}(\mathscr{M})=\varnothing$ for all $k \in\{2, \ldots, d\}$ the only extension $\mathscr{M}^{\prime \prime}$ of $\mathscr{M}$ with $\mathscr{M}^{\prime \prime}<\mathscr{M}$ is the trivial extension given by $\mathscr{M}^{\prime \prime}(f)=0$ (i.e., $f$ is a loop in $\mathscr{M}^{\prime \prime}$ ). Assume on the contrary that there is any extension $\mathscr{M}^{\prime} \in \mathscr{E}(\mathscr{M} \backslash f)$ with $\mathscr{M}^{\prime \prime}<\mathscr{M}^{\prime}<\mathscr{M}$ with $\chi_{\mathscr{H}^{\prime}}(f, \lambda)=0$ for a suitable

$$
\lambda \in \Lambda(E-\{f\}, d-1)
$$

Repeated application of Claim 3 yields $\chi_{\mathscr{N}}(f)=0$ which contradicts the assumption. This completes the proof of Theorem 2.7. 
Combining Theorems 2.7 and 2.6 we immediately obtain:

Corollary 2.8. If a uniform oriented matroid $\mathscr{M}$ of rank 4 contains an element $f$ such that $M_{\mu} t_{f}(\mathscr{M})$ is empty, then $\mathscr{M}$ is an isolated element in $\mathscr{E}(\mathscr{M} \backslash f)$.

We finally present a rank 4 oriented matroid $R(20)$ on $E:=\{1, \ldots, 20\}$ such that $\operatorname{Mut}_{2}(R(20))=\varnothing$. A detailed description of the underlying construction
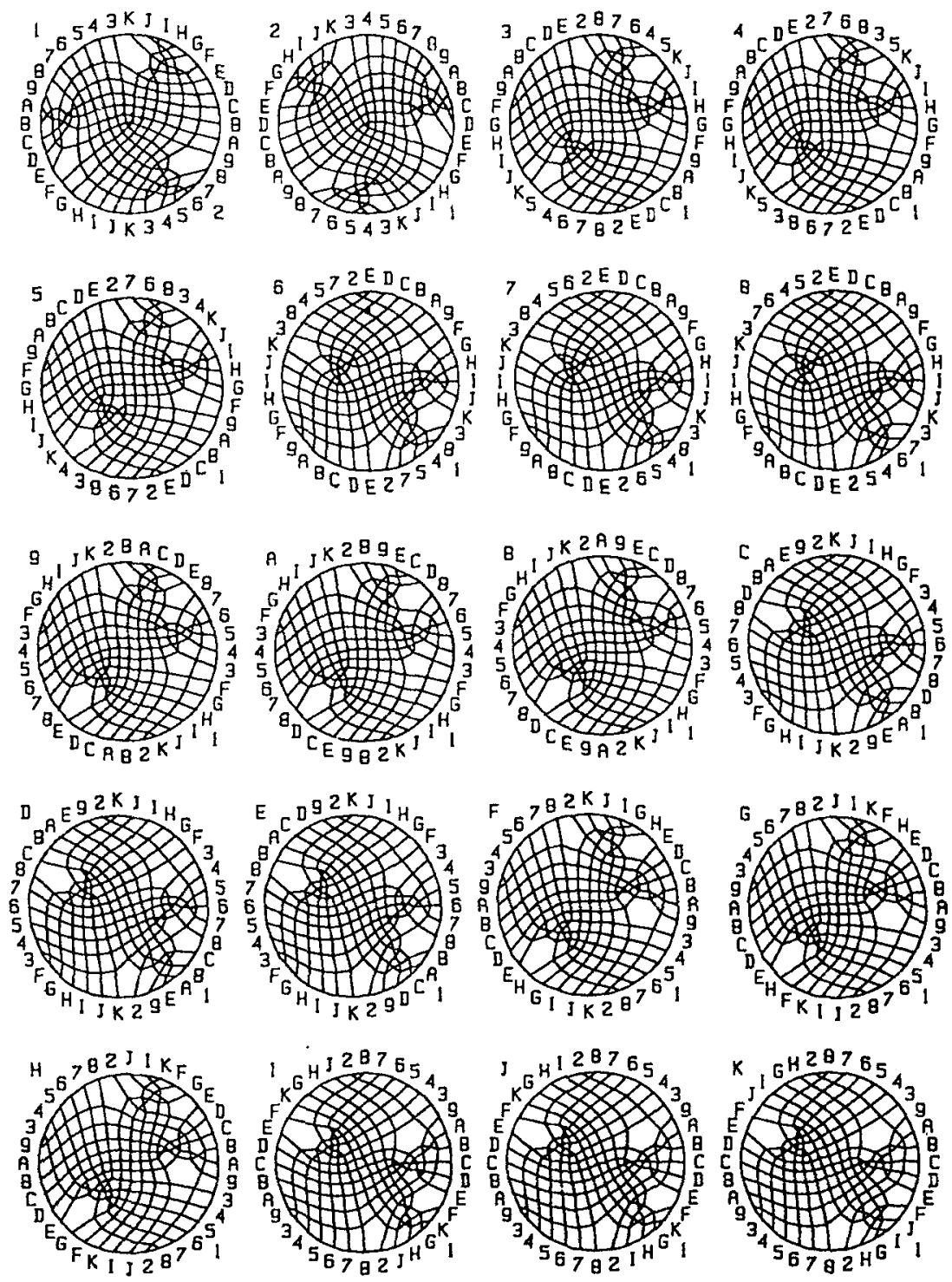

Fig. 2. The 20 one-element contractions of $R(20)$. 
methods is given in [10]. There rank-preserving composition methods of oriented matroids are described in order to derive oriented matroids with a small number of mutations.

Here we restrict ourselves to giving a combinatorial description of $R(20)$. Figure 2 contains pictures of the pseudoline arrangements of the 20 contractions of $R(20)$. The elements of $R(20)$ are labeled $1, \ldots, 9, A, \ldots, K$. In every picture the label of the contracted element is marked at the upper left corner and the chosen line at infinity is marked at the lower right corner. The information given in Fig. 2 is sufficient to determine the reorientation class of $R(20)$.

The oriented matroid $R(20)$ is constructed by taking the connected sum as defined in [10] of four copies of the unique rank 4 oriented matroid with eight elements and only seven mutations (see [11] and [4]). $R(20)$ has only 16 mutations:

$$
\begin{aligned}
\operatorname{Mut}(R(20))= & \{(1,3,9, F),(1,4,6,8),(1, A, C, E),(1, G, I, K),(3,4,5,8),(3,6,7,8) \\
& (4,5,6,7),(4,5, A, B),(6,7, G, H),(9, A, B, E),(9, C, D, E) \\
& (A, B, C, D),(C, D, I, J),(F, G, H, K),(F, I, J, K),(G, H, I, J)\}
\end{aligned}
$$

The mutations of $R(20)$ can be easily read off from Fig. 2. A quadruple $\left(\lambda_{1}, \lambda_{2}, \lambda_{3}, \lambda_{4}\right)$ is a mutation of $R(20)$ if for every $i=1, \ldots, 4$ the lines

$$
\left(\lambda_{1}, \ldots, \hat{\lambda}_{i}, \ldots, \lambda_{4}\right)
$$

are adjacent to a triangular cell in the pseudoline picture of the contraction $R(20) / \lambda_{i}$. We obtain:

Theorem 2.9. $R(20)$ is an isolated element in the extension space $\mathscr{E}(R(20) \backslash 2)$.

Proof. Observe that $M u t_{2}(R(20))=\varnothing$. Applying Corollary 2.8 immediately gives the desired result.

\section{References}

1. L. J. Billera, M. M. Kapranov, and B. Sturmfels: Cellular strings on polytopes. Preprint, Cornell University, 1991.

2. A. Björner, M. Las Vergnas, B. Sturmfels, N. White, and G. M. Ziegler: Oriented Matroids. Encyclopedia of Mathematics and Its Applications, Vol. 46. Cambridge University Press, Cambridge, 1993.

3. R. G. Bland: A combinatorial abstraction of linear programming. J. Combin. Theory Ser. B 23 (1977), 33-57.

4. J. Bokowski and J. Richter-Gebert: On the classification of non-realizable oriented matroids, Part II: properties. Preprint, TH-Darmstadt, 1990.

5. J. Bokowski and B. Sturmfels: Computational Synthetic Geometry. Lecture Notes in Mathematics, Vol. 1355. Springer-Verlag. Berlin, 1989.

6. J. Folkman and J. Lawrence: Oriented matroids. J. Combin. Theory Ser. B 25 (1978), 199-238.

7. K. Fukuda: Oriented Matroid Programming. Ph.D. Thesis, University of Waterloo, 1982. 
8. M. Las Vergnas: Extensions ponctuelles d'une géométrie combinatoire orienté. In: Problèmes combinatoires et théorie des graphes (Actes Coll., Orsay, 1976). Colloques internationaux, No. 260. C.N.R.S., 1978, pp. 265-270.

9. A. Mandel: Topology of Oriented Matroids. Ph.D. Thesis, University of Waterloo, 1982.

10. J. Richter-Gebert: Oriented matroids with few mutations. Discrete Comput. Geom., this issue, pp. 251-269.

11. J. P. Roudneff and B. Sturmfels: Simplicial cells in arrangements and mutations of oriented matroids. Geom. Dedicata 27 (1988), 153-170.

12. B. Sturmfels and G. Ziegler: Extension spaces of oriented matroids. Discrete Comput. Geom. 10 (1993), 23-45.

Received December 23, 1991, and in revised form February 18, 1993. 